\title{
Rotation-Vibration Interaction in the Microwave Spectrum of Fulminic Acid, HCNO*
}

\author{
Hans Karl Bodenseh \\ Lehrstuhl für Physikalische Chemie, Universität Ulm \\ and Manfred WinNewisser \\ Institut für Physikalische Chemie, Universität Kiel \\ (Z. Naturforsch. 24 a, 1966—1972 [1969]; received 2 August 1969)
}

\begin{abstract}
Rotational transitions in the microwave spectrum $(20-46 \mathrm{GHz})$ of HCNO have been measured and assigned to seven excited vibrational states. The direct $l$-type doubling transitions of the $\Pi$-component of the vibrational levels $v_{5}=3$ have been measured and analyzed, yielding the following values:

$$
\begin{aligned}
q_{3 \times 5}^{(0)} & =27.0920 \pm 1.8 \cdot 10^{-4} \mathrm{MHz} \\
E_{\Pi}-E_{\Phi} & =\Delta \cong 65 \mathrm{~cm}^{-1} .
\end{aligned}
$$
\end{abstract}

In a previous communication we reported the microwave spectrum of the linear molecule HCNO, its structure and $l$-type doubling for the $v_{4}=1$ and $v_{5}=1 \Pi$-states ${ }^{1}$. This paper is concerned with the assignment and the theoretical analysis of the observed transitions of HCNO molecules in additional excited vibrational states. The transitions measured are $J=0 \rightarrow 1$ and $J=1 \rightarrow 2$ rotational transitions in the 20 to $46 \mathrm{GHz}$ region, so that the vibrational satellites observed arise either from $\Sigma$-states with $l=0$, or $\Pi$-states with $l=1$. In addition, the direct $l$-type doubling series for the $\Pi$-state $(l=1)$ of the $3 v_{5}$-vibrational levels has been measured and analysed.

The spectrometer and related experimental aspects of the measurements are described in Ref. ${ }^{1}$. The yield from the preparation described there has been considerably increased, so that visible crystals of pure HCNO can be obtained.

The high resolution infrared measurements of WINNEWISSER and WINNEWISSER ${ }^{2}$ on the $v_{1}$-band have shown conclusively that this substance, whose microwave spectrum is reported here an in Ref. ${ }^{1}$, is identical with the compound prepared by BECK and FELDL ${ }^{3}$ for low resolution infrared scans.

Reprint requests to Dr. M. WINNEWISSER, Institut für Physikalische Chemie der Universität Kiel, D-2300 Kiel, Olshausenstraße.

* Presented in part at the Chemiedozententagung 1968, April $3-5$ in Hamburg, and at the 23rd Symposium on Molecular Structure and Spectroscopy at the Ohio State University, Columbus, Ohio, September 3-7, 1968.

1 M. WinNewisser and H. K. Bodenseh, Z. Naturforsch. 22 a, 1724 [1967].

\section{Rotation-Vibration Interaction in a Linear Molecule}

\author{
a) Rotational Constant $B_{v}$
}

The rotational constant of a linear molecule in a given vibrational state may be expanded in the form

$$
\begin{aligned}
B_{v}=B_{\mathrm{e}} & -\sum_{i} \alpha_{i}\left(v_{i}+d_{i} / 2\right) \\
& +\sum_{i, j} \gamma_{i j}\left(v_{i}+d_{i} / 2\right)\left(v_{j}+d_{j} / 2\right)+\sum_{i, j} \gamma_{l_{i} l_{j}} l_{i} l_{j} .
\end{aligned}
$$

The factors $\gamma_{i j}$ and $\gamma_{l_{i} l_{j}}$ are in general small quantities for which there are few known examples. MAKI and BLAINE ${ }^{4}$ report various $\gamma_{i j}$ for $\mathrm{HCN}$ and the relatively large value of $\gamma_{l l} \equiv \gamma_{l_{2} l_{2}}=-6.3 \mathrm{MHz}$ which is reduced to $-2.7 \mathrm{MHz}$ already for $\mathrm{DCN}^{5}$. In the case of OCS the value $-0.059 \mathrm{MHz}$ was determined for $\gamma_{22}{ }^{6}$. For small values of $v_{i}$, that is 0,1 or 2 , and the corresponding values of $l_{i}$ (only $l=0$ and 1 are possible in the rotational transitions $J=0 \rightarrow 1$ and $J=1 \rightarrow 2$ ), the contributions of the terms involving $\gamma$ should be less than $10 \mathrm{MHz}$. Thus deviations from the simplified relation

$$
B_{v}=B_{\mathrm{e}}-\sum_{i} \alpha_{i}\left(v_{i}+d_{i} / 2\right)
$$

2 B. P. WinNewisser and M. WinNewisser, J. Mol. Spectry. 29, 505 [1969].

3 W. BeCK and K. Feldl, Angew. Chem. 78, 746 [1966].

4 A. G. Maki, Jr., and L. R. Blaine, J. Mol. Spectry. 12, 45 [1964].

5 A. G. Maki, Jr., and D. R. Lide, Jr., J. Chem. Phys. 47, 3206 [1967].

6 Y. Morino and C. Matsumara, Bull. Chem. Soc. Japan 40, 1095 [1967]. 
larger than $10 \mathrm{MHz}$ can be attributed to anharmonic resonance interactions involving the given vibrational state $v$. The multiplicity of low-lying vibrational levels in HCNO makes resonances likely, but a full interpretation will be dependent on the determination of the $\gamma$ 's from data obtained from molecules in higher excited vibrational states.

\section{b) l-Type Phenomena}

As a linear four-atomic molecule, fulminic acid, HCNO, may be expected to exhibit both

1 ) vibrational $l$-type doubling and $l$-type resonance, and

2 ) rotational $l$-type doubling and $l$-type resonance, as defined by AMAT and Nielsen ${ }^{7}$. These phenomena can be accounted for by matrix elements of the transformed second order Hamiltonian, $H^{(2)^{\prime}}$, which are off diagonal in $l$.

The effects known as "vibrational" $l$-type doubling and resonance can be calculated from matrix elements of the form

$\left(v_{\mathrm{t}}, l_{\mathrm{t}} ; v_{\mathrm{t}^{\prime}}, l_{\mathrm{t}^{\prime}} ; \ldots\left|H^{(2)^{\prime}}\right| v_{\mathrm{t}}, l_{\mathrm{t}} \pm 2 ; v_{\mathrm{t}^{\prime}}, l_{\mathrm{t}^{\prime}} \mp 2 ; \ldots\right)$

which introduce contributions to the vibrational energy which are independent of the rotational quantum number $J$. Such effects are to be observed in states involving the excitation of two bending modes, such as $v_{4}+v_{5}$, where the $\Sigma$-state, $000(11)^{0}$, is split into two components $\Sigma^{+}$and $\Sigma^{-}$separated by the vibrational constant $r_{45}$ defined in Ref. ${ }^{7}$.

Interactions known as "rotational" $l$-type doubling and resonance are due to matrix elements of the form

$$
\left(v_{\mathrm{t}}, l_{\mathrm{t}} ; \ldots\left|H^{(2)^{\prime}}\right| v_{\mathrm{t}}, l_{\mathrm{t}} \pm 2 ; \ldots\right)
$$

and introduce a $J(J+1)$ dependent splitting of otherwise degenerate levels ( $l$-type doubling) or a higher order shifting or a splitting of interacting levels which may or may not be otherwise degenerate ( $l$-type resonance). These effects must be considered for states such as $v_{5}, 2 v_{5}$ and $3 v_{5}$.

\section{c) l-Type Doubling for the Case $v_{\mathrm{t}}=3$}

The third excited state of the twofold degenerate mode $\nu_{5}$ is composed of a $\Pi$ - and a $\Phi$-level, with the quantum-number $l$ assuming the values 1 and 3 , respectively. The rotational levels of the $\Pi$-state exhibit $l$-type doubling, thus giving rise to doublet transitions in the microwave region analogous to the transitions measured for the $v_{5}=1$ state ${ }^{1}$.

However, an analysis of the observational data cannot be carried out by simply using the wellknown relation derived for the $v_{\mathrm{t}}=1 l$-type doubling of $\Pi$-states

$$
f=q J(J+1),
$$

because the $0000^{0} 3^{1}$ and $0000^{0} 3^{3}$ states are connected by the same matrix elements given above [Eq. (4)], which also link the levels $3^{+1}$ and $3^{-1}$ following the AMAT-NIELSEN notation ${ }^{7}$.

The matrix elements connecting the degenerate levels $3^{+1}$ and $3^{-1}$ cause a splitting of the rotational energy levels, while the matrix elements linking the $3^{ \pm 3}$ and $3^{ \pm 1}$ states result in

1) a shifting of the vibration-rotational levels,

2 ) an alteration of the $\Pi$-state $l$-type splitting, and

3 ) a splitting of the $\Phi$-state rotational levels.

This interaction of the four states is a case of $l$-type resonance.

The secular determinant for the interaction between $\Pi$ and $\Phi$ levels for $v_{5}=3$

\begin{tabular}{|c|c|c|c|c|}
\hline & $l=+3$ & $l=+1$ & $l=-1$ & $l=-3$ \\
\hline$l=+3$ & $E_{3}{ }^{0}-\lambda$ & $H_{31}$ & 0 & 0 \\
\hline$l=+1$ & $H_{31}$ & $E_{1}^{0}-\lambda$ & $H_{11}$ & 0 \\
\hline$l=-1$ & 0 & $H_{11}$ & $E_{1}{ }^{0}-\lambda$ & $H_{31}$ \\
\hline$l=-3$ & 0 & 0 & $H_{31}$ & $E_{3}{ }^{0}-\lambda$ \\
\hline
\end{tabular}
is given by:

By forming the linear combinations of the initial wave functions

$$
{\psi_{3}}^{+}=\frac{1}{\sqrt{2}}\left(\psi_{+3}+\psi_{-3}\right) ; \quad{\psi_{3}}^{-}=\frac{1}{\sqrt{2}}\left(\psi_{+3}-\psi_{-3}\right) ; \quad{\psi_{1}}^{+}=\frac{1}{\sqrt{2}}\left(\psi_{+1}+\psi_{-1}\right) ; \quad \psi_{1}^{-}=\frac{1}{\sqrt{2}}\left(\psi_{+1}-\psi_{-1}\right) ;
$$

\footnotetext{
7 G. Amat and H. H. Nielsen, J. Mol. Spectry. 2, 152 [1958].
} 
the determinant may be written in the following form:

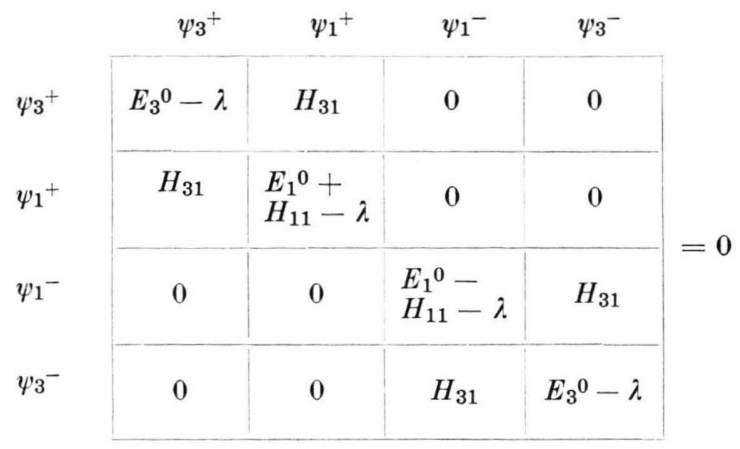

yielding as solutions the perturbed energies for the $\Pi$ - and $\Phi$-states:

$E_{3}^{+}=E_{3}^{0}-\frac{1}{2} \Delta+\frac{1}{2} H_{11}+\frac{1}{2}\left[\left(\Delta-H_{11}\right)^{2}+4 H_{31}^{2}\right]^{1 / 2}$, $E_{1}^{+}=E_{1}^{0}+\frac{1}{2} \Delta+\frac{1}{2} H_{11}-\frac{1}{2}\left[\left(\Delta-H_{11}\right)^{2}+4 H_{31}^{2}\right]^{1 / 2}$, $E_{1}^{-}=E_{1}^{0}+\frac{1}{2} \Delta-\frac{1}{2} H_{11}-\frac{1}{2}\left[\left(\Delta+H_{11}\right)^{2}+4 H_{31}^{2}\right]^{1 / 2}$, $E_{3}^{-}=E_{3}^{0}-\frac{1}{2} \Delta-\frac{1}{2} H_{11}+\frac{1}{2}\left[\left(\Delta+H_{11}\right)^{2}+4 H_{31}^{2}\right]^{1 / 2}$

where $\Delta$ is defined as the energy difference between the unperturbed $\Phi$ - and $\Pi$-states:

$$
\Delta=E_{\Phi}^{0}-E_{\Pi}^{0}=E_{3}^{0}-E_{1}^{0} .
$$

From the general form of the matrix elements as given by NIELSEN and SHAFFER ${ }^{8}$

$$
\begin{aligned}
\left(v_{\mathrm{t}}, l_{t}\left|H^{(2)^{\prime}}\right| v_{\mathrm{t}}, l_{\mathrm{t}} \pm 2\right)=\frac{1}{4} q\left[\left(v_{\mathrm{t}} \mp l_{\mathrm{t}}\right)\left(v_{\mathrm{t}} \pm l_{\mathrm{t}}+2\right)\right]^{1 / 2} \\
\cdot\left[J(J+1)-l_{\mathrm{t}}\left(l_{\mathrm{t}} \pm 1\right)\right]^{1 / 2} \\
\cdot\left[J(J+1)-\left(l_{\mathrm{t}} \pm 1\right)\left(l_{\mathrm{t}} \pm 2\right)\right]^{1 / 2}
\end{aligned}
$$

the matrix elements for this case may be evaluated:

$H_{11}=q J(J+1)$,

$\left.H_{31}=q(\sqrt{3} / 2)\left[J^{2}(J+1)^{2}-8 J(J+1)+12\right)\right]^{1 / 2}$

with

$$
q=q^{(0)}-q^{(1)} J(J+1)+q^{(2)}[J(J+1)]^{2} \ldots
$$

as defined in Ref. ${ }^{1}$.

Taking $l$-type resonance into acount will therefore yield as doublet separation of the $\Pi$-levels the following frequency expression:

$$
\begin{aligned}
f=q \cdot J(J+1) & -\frac{1}{2}\left\{[\Delta+q J(J+1)]^{2}+3 q^{2}\left[J^{2}(J+1)^{2}-8 J(J+1)+12\right]\right\}^{1 / 2} \\
& +\frac{1}{2}\left\{[\Delta-q J(J+1)]^{2}+3 q^{2}\left[J^{2}(J+1)^{2}-8 J(J+1)+12\right]\right\}^{1 / 2} .
\end{aligned}
$$

\section{d) Anharmonic Force Constants}

The terms in the second order transformed Hamiltonian $H^{(2)^{\prime}}$ which determine the vibrational $l$ type interaction as well as those leading to anharmonic resonance interactions are expressed in terms of the harmonic frequencies and the cubic and quartic force constants ${ }^{9}$.

In general there are as many cubic coefficients as there are possible combinations of any three normal coordinates, but the requirement that the potential function be symmetric with respect to all symmetry operations requires that any coefficient of an odd product of normal coordinates which are not totally symmetric be zero. For a linear molecule with two bending normal coordinates, $Q_{4}$ and $Q_{5}$, and symmetry $\mathrm{C}_{\infty \mathrm{v}}$ this means that the only nonzero

8 H. H. Nielsen and W. H. Shaffer, J. Chem. Phys. 11, 140 [1943]. Erratum: Phys. Rev. 75, 1961 [1949]. cubic force constants involving $Q_{4}$ and (or) $Q_{5}$ are $k_{n 45}, k_{n 44}$, and $k_{n \check{5} 5}$, where $n=1,2$ or 3 .

These force constants are parameters describing possible first-order Fermi interactions involving the bending modes.

Second order anharmonic resonance such as the Darling-Dennison type interaction discussed below involve quartic as well as cubic terms. The quartic force constants are subject to the same considerations of the symmetry of the potential function as was the case for the cubic force constants. The only quartic constant linking the two bending modes directly is the term $k_{4455}$. Since the other nonzero quartic force constants are not necessary for the anharmonic resonance interactions discussed below in the second order approximation they are omitted here.

\footnotetext{
9 H. H. Nielsen, Handbuch der Physik, Band XXXVII/1,
} Atome III-Moleküle I, Springer-Verlag, Berlin 1959. 


\begin{tabular}{|c|c|c|c|c|c|c|c|c|c|}
\hline \multirow[t]{2}{*}{$\begin{array}{l}\text { Rotational } \\
\text { transition }\end{array}$} & \multicolumn{5}{|c|}{$\begin{array}{c}\text { Vibrational } \\
\text { state }\end{array}$} & \multirow[t]{2}{*}{$\begin{array}{l}\text { Symmetry } \\
\text { species }\end{array}$} & \multirow{2}{*}{$\begin{array}{c}\text { Measured } \\
\text { frequency } \\
f(\mathrm{MHz})\end{array}$} & \multicolumn{2}{|c|}{ Spectroscopic constanst } \\
\hline & $v_{1}$ & $v_{2}$ & $v_{3}$ & & $v_{5}^{l}$ & & & $B_{v}(\mathrm{MHz})$ & $\alpha_{v}(\mathrm{MHz})$ \\
\hline \multirow[t]{6}{*}{$J=0 \rightarrow 1$} & 0 & 0 & 0 & $0^{0}$ & $0^{0}$ & $\Sigma^{+}$ & $22938.07 \pm 0.05$ & 11469.04 & $\mathrm{a}$ \\
\hline & 0 & 0 & 1 & $0^{0}$ & $0^{0}$ & $\Sigma^{+}$ & $22866.38 \pm 0.1$ & 11433,19 & $+35.85^{\mathrm{a}}$ \\
\hline & 0 & 0 & 0 & $2^{0}$ & $0^{0}$ & $\bar{\Sigma}^{+}$ & $22996.56+0.1$ & 11498.28 & $-29.24^{b}$ \\
\hline & 0 & 0 & 0 & $0^{0}$ & $2^{0}$ & $\bar{\Sigma}^{+}$ & $23031.45 \mp 0.1$ & 11515.73 & $-46.69^{b}$ \\
\hline & 0 & 0 & 0 & (1 & $1)^{0}$ & $\bar{\Sigma}^{-} l_{\mathrm{c}}$ & $23026.71 \pm 0,1$ & 11513.36 & $-44.32^{b}$ \\
\hline & 0 & 0 & 0 & $(1$ & 1) 0 & $\bar{\Sigma}+\}^{\mathrm{c}}$ & $23029.33 \pm 0.1$ & 11514.67 & $-45.63^{b}$ \\
\hline \multirow[t]{7}{*}{$J=1 \rightarrow 2$} & 0 & 0 & 0 & $0^{0}$ & $0^{0}$ & $\Sigma^{+}$ & $45876.09 \pm 0.05$ & 11469.02 & a \\
\hline & 0 & 0 & 0 & $1_{1}^{1}$ & $0^{0}+$ & $\Pi$ & $45886.41 \pm 0.1$ & 11483.44 & $-14.42^{\mathrm{a}}$ \\
\hline & $\begin{array}{l}0 \\
0\end{array}$ & $\begin{array}{l}0 \\
0\end{array}$ & $\begin{array}{l}0 \\
0\end{array}$ & $\begin{array}{l}1_{2}^{1} \\
0^{0}\end{array}$ & $\begin{array}{l}\left.0^{0}\right\} \\
\left.1_{1}^{1}\right\}\end{array}$ & & $\begin{array}{l}45981.08 \pm 0.1 \\
45927.51 \pm 0.05\end{array}$ & & \\
\hline & 0 & 0 & 0 & $0^{0}$ & $\left.\mathbf{1}_{2}^{1}\right\}$ & II & $46065.97 \pm 0.05$ & 11499.19 & $-30.17^{a}$ \\
\hline & 0 & 0 & 0 & $0^{0}$ & $2^{0}$ & $\Sigma^{+}$ & $46062.82 \pm 0.1$ & 11515.71 & $-46.69^{b}$ \\
\hline & 0 & 0 & 0 & $(1$ & $1)^{0}$ & $\Sigma^{-} l_{\mathrm{c}}$ & $46053.43 \pm 0.1$ & 11513.36 & $-44.34^{b}$ \\
\hline & 0 & 0 & 0 & $(1$ & $1)^{0}$ & $\Sigma+\}^{c}$ & $46058.47 \pm 0.1$ & 11514.62 & $-45.60^{b}$ \\
\hline
\end{tabular}

${ }^{\mathrm{a}}$ See Ref. ${ }^{1} .{ }^{\mathrm{b}}$ This work. $-{ }^{\mathrm{c}}$ The given symmetry designation + or - of these two $\Sigma$-states is arbitrary since the microwave measurements do not allow this to be determined.

Table 1. Measured line frequencies and spectroscopic constants for the assigned vibrational states of $\mathrm{H}^{12} \mathrm{C}^{14} \mathrm{~N}^{16} \mathrm{O}$.

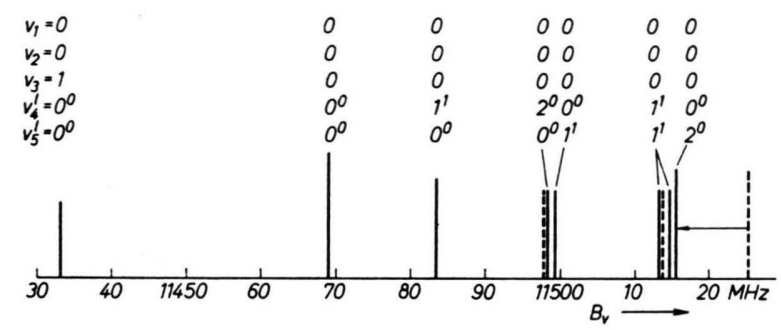

Fig. 1. Measured (solid lines) and calculated (dotted lines) rotational constants of different vibrational states of the molecule $\mathrm{H}^{12} \mathrm{C}^{14} \mathrm{~N}^{16} \mathrm{O}$ from the rotational transitions

$$
J=0 \rightarrow 1 \text { and } J=1 \rightarrow 2 .
$$

\section{Experimental Results and Discussion}

\section{a) Assignment of Vibrational Satellites in the $R$-Branch}

The rotational constants $B_{v}$ and the rotationvibration interaction constants $\boldsymbol{\alpha}_{v}$ for all of the assigned vibrational satellites of $\mathrm{H}^{12} \mathrm{C}^{14} \mathrm{~N}^{16} \mathrm{O}$ are listed in Table 1. To clarify the following discussion the rotational constants are plotted in Fig. 1, with positions expected according to Eq. (2) represented by the dotted lines.

The rotational transition in the first excited state of the lowest stretching mode, $v_{3}$, (far left in Fig. 1) could easily be identified due to the positive value of $\alpha$ and the relative intensity of the absorption line. To higher frequencies are found first the ground state transition, and then the first excited states of the two bending modes. Since these are
$\Pi$-states, they appear only in the $J=1 \rightarrow 2$ transition in the form of $l$-type doublets. The $l$-type doublet splitting and the relative intensity make the assignment of these transitions unique. Next to the rotational constant for $0000^{0} 1^{1}$ lies the $B_{v}$ value of the state $0002^{\circ} 0^{\circ}$, at a distance almost exactly $2 \alpha_{4}$ from the ground state, as expected. The apparent triplet near $11515.0 \mathrm{MHz}$ was more difficult to assign. Fig. 2 shows the $J=0 \rightarrow 1$ absorption of these three lines which all arise from $\Sigma$-vibrational states.

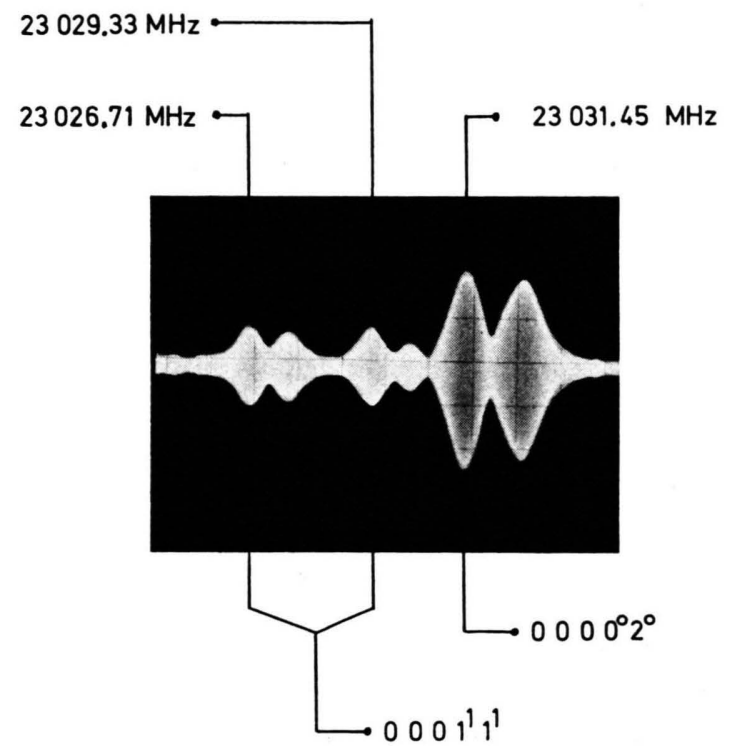

Fig. 2. Oscilloscope trace of the rotational transition $J=0 \rightarrow 1$ of $\mathrm{H}^{12} \mathrm{C}^{14} \mathrm{~N}^{16} \mathrm{O}$ molecules in the vibrational states $000(11)^{0}$ and $0000^{\circ} 2^{0}$ and their corresponding Stark-satellites. 
Measurements taken at various temperatures indicated that the two weaker lines form a doublet, whose relative intensity stayed the same over the temperature range $+30^{\circ} \mathrm{C}$ to $-70{ }^{\circ} \mathrm{C}$ within the accuracy of the intensity measurement, whereas the intensity of the third line relative to the doublet is strongly dependent on the temperature. Thus the doublet must be assigned to two levels which lie very close to one another, and the third line to a level which lies $200 \pm 10 \mathrm{~cm}^{-1}$ lower than the doublet levels, according to the intensity measurements.

Identification of the levels observed can best be carried out with the help of the energy level diagram shown in Fig. 3. This diagram shows all vi-

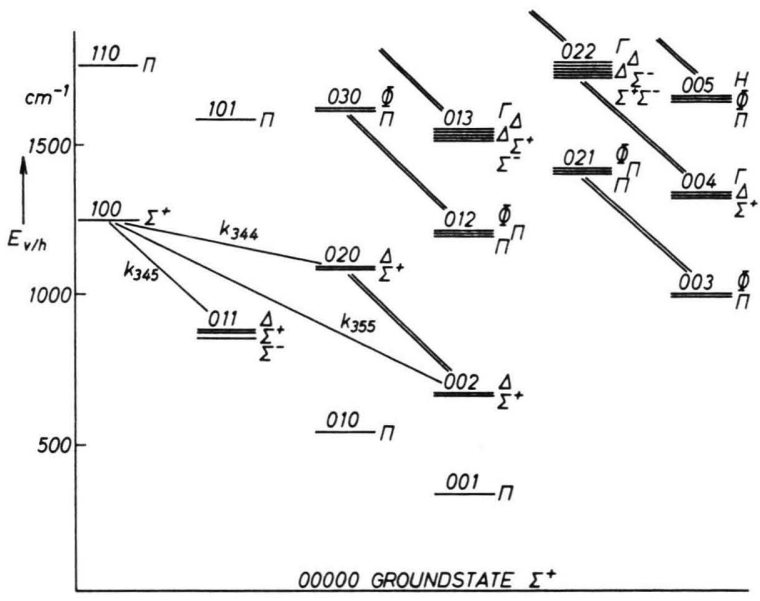

Fig. 3. Lower region of the vibrational energy level diagram of the linear molecule HCNO. $v_{1}, v_{2}$ and all $l$ 's have been omitted in labelling the levels. The level $v_{5}=1$ has been estimated from microwave data.

brational levels of HCNO lying below $1800 \mathrm{~cm}^{-1}$, based on the presently available but still rather limited infrared data ${ }^{2,3}$. Single lines connect states of the same symmetry species between which Fermi resonance is possible, with the relevant cubic force constants indicated. Thus it can be seen that the first excited states of $v_{4}$ and $v_{5}$ can interact neither with each other nor with any other nearby level, since there is no force constant connecting them. The first level with which a resonance is possible is nearly $1600 \mathrm{~cm}^{-1}$ away, so that it can be neglected. Since these $\Pi$-levels are unperturbed, the $\alpha$ values from these levels may be used to calculate the expected $\alpha$ values for combination states and higher excited states of $v_{4}$ and $v_{5}$. The $\alpha$ values calculated in this way for the $2 v_{4}, 2 v_{5}$ and $\left(v_{4}+v_{5}\right)$-states are given in Table 2. The calculated $\alpha$ for the $\left(v_{4}+v_{5}\right)$-state

\begin{tabular}{|c|c|c|c|c|}
\hline $\begin{array}{c}\text { Vibrational } \\
\text { state } \\
v_{1} v_{2} v_{3} v_{4}^{l} v_{5}^{l}\end{array}$ & $\begin{array}{c}\alpha_{v} \text { (meas.) } \\
(\mathrm{MHz})\end{array}$ & $\begin{array}{c}\alpha_{v} \text { (calc.) } \\
(\mathrm{MHz})\end{array}$ & $\begin{array}{c}\alpha_{v} \text { (meas.) } \\
-\alpha_{v}(\text { calc. }) \\
(\mathrm{MHz})\end{array}$ & $\begin{array}{c}\text { Deviation } \\
\%\end{array}$ \\
\hline $0002^{0} 0^{0}$ & -29.24 & -28.84 & -0.4 & -1.4 \\
\hline $0000^{0} 2^{0}$ & -46.69 & -60.34 & +13.65 & +22.6 \\
\hline $0000\left(\begin{array}{lll}1 & 1\end{array}\right)^{0}$ & $-44.98^{\mathrm{a}}$ & $-44.59^{a}$ & -0.39 & -0.9 \\
\hline
\end{tabular}

a Average values of the two components of the $\left(\Sigma^{-}, \Sigma^{+}\right)$ doublet.

Table 2. Calculated and measured values of the vibrationrotation-interaction constant $\alpha_{v}$ for the $v_{4}$ and $v_{5}$ vibrational satellites of $\mathrm{H}^{12} \mathrm{C}^{14} \mathrm{~N}^{16} \mathrm{O}$.

leads to a rotational constant which agrees very well with the average value of the two components of the doublet mentioned above.

The $\left(v_{4}+v_{5}\right)$ combination state consists of the $\Delta$-state $000(11)^{2}$, which will only appear in the $J=2 \rightarrow 3$ or higher transition, and which is degenerate in the harmonic approximation, and the state $000(11)^{0}$ which has a $\Sigma^{+}$and a $\Sigma^{-}$component. These two components are split by vibrational $l$-type doubling as described above. The derivation of the doubling term does not necessarily lead to an observable difference in the rotational constants. However, a shift of the $\Sigma^{+}$level could arise through a Fermi resonance interaction with the level $0010^{\circ} 0^{0}$. The very small value of the difference of the rotational constants is evidence that such a resonance interaction, if present, must be weak.

The third member of this group of lines, since it comes from a vibrational level lying $200 \mathrm{~cm}^{-1}$ lower, must be assigned to the $\Sigma$-component of the $2 v_{5}$ level, $0000^{0} 2^{0}$.

A comparison with the calculated value of the rotational constant for this level shows that the observed value deviates by $13 \mathrm{MHz}$, or $23 \%$ from the $\alpha$ value for $2 v_{5}$. This difference indicates a contribution of the term $\gamma_{55}\left(v_{5}+1\right)^{2}$, but the large magnitude suggests that contributions due to resonance interactions are involved. However, the nearest energy level with which Fermi resonance is possible, $v_{3}$, is $600 \mathrm{~cm}^{-1}$ away, so that a strong interaction is not likely. In addition, the cubic force constant connecting these two levels would be $k_{355}$; if $k_{344}$ is of a similar magnitude, the corresponding resonance of $v_{3}$ with $2 v_{4}$ should be even larger, since the latter lies much closer to $v_{3}$. The fact that the $2 v_{4}$ rotational constant is unshifted is therefore further reason to eliminate Fermi resonance with $v_{3}$ as the principle source of a shift in the $B$ value of $2 v_{5}$. 
Since no other first order resonance perturbations are possible, it is necessary to seek an explanation of this shift in a perturbation of higher order. The most likely second order perturbation is one of the type first interpreted by DARLING and DENNISON ${ }^{\mathbf{1 0}}$. This type of interaction occurs due to off-diagonal matrix elements of the second order vibrational Hamiltonian of the form

$$
\left(v_{\mathrm{t}}, v_{\mathrm{t}^{\prime}}, v_{\mathrm{t}^{\prime \prime}}, \ldots\left|H^{(2)^{\prime}}\right| v_{\mathrm{t}} \pm 2, v_{\mathrm{t}^{\prime}} \mp 2, v_{\mathrm{t}^{\prime \prime}}, \ldots\right)
$$

when the two levels involved have nearly the same energy. In the case of HCNO, in which both of the vibrational modes involved are degenerate, the matrix elements have the form ${ }^{11}$

$$
\begin{aligned}
\left(v_{4}, l_{4} ; v_{5}, l_{5} ; v_{n}, \ldots\left|H^{(2)^{\prime}}\right| v_{4} \pm 2, l_{4} ; v_{5} \mp 2, l_{5} ; v_{n}, \ldots\right) \\
=\frac{1}{4} \gamma\left[\left(v_{4}+l_{4}+1 \pm 1\right)\left(v_{4}-l_{4}+1 \pm 1\right)\right. \\
\left.\left(v_{5}+l_{5}+1 \mp 1\right)\left(v_{5}-l_{5}+1 \mp 1\right)\right]^{1 / 2}
\end{aligned}
$$

where $\gamma$ is a function of the quartic force constant $k_{4455}$ and some of the cubic force constant products $\left(k_{n 45}\right)^{2}$ and $\left(k_{n 44}\right)\left(k_{n 55}\right)$ with $n=1,2,3^{9}$. Levels in Fig. 3 which could exhibit this type of resonance are connected by double lines.

As can be seen from Fig. 3, there are, in all, five vibrational levels interacting through the constants $k_{344}, k_{355}, r_{45}$, and $\gamma$. If the rotational energy is also considered, the resulting $l$-type doubling raises the number of levels and thus the order of the problem to 11. A numerical calculation of the perturbed and unperturbed energy levels is thus not possible with the limited amount of data presently available. Analogous data has been obtained for the deuterofulminic acid, DCNO ${ }^{12}$.

\section{b) Analysis of the Observed l-Type Doubling Transitions for the $0000^{0} 3^{1}$ Vibrational State of $\mathrm{H}^{12} \mathrm{C}^{14} \mathrm{~N}^{16} \mathrm{O}$}

Thirteen transitions of this type have been observed in the frequency range 10 to $35 \mathrm{GHz}$ with $J=13$ to $J=25$, and are listed in Table 3 .

In the least squares analysis used in fitting the data, the residual equations were derived from $\mathrm{Eq}$. (14) and the constants $q^{(0)}, q^{(1)}$ and $\Delta$ were determined independently. An attempt to evaluate $q_{5}{ }^{(2)}$ failed because of the small magnitude of the constant $q^{(2)}$ (for $v_{5}=1, q_{5}^{(2)}=1 \cdot 10^{-9} \mathrm{MHz}$ ) and the

10 B. T. Darling and D. M. Dennison, Phys. Rev. 57, 128 [1940].

11 W. H. Shaffer, Rev. Mod. Phys. 16, 245 [1944].

\begin{tabular}{cccc}
\hline$J$ & $\begin{array}{c}\text { Observed } \\
\text { frequencies } \\
(\mathrm{MHz})\end{array}$ & $\begin{array}{c}\text { Calculated } \\
\text { frequencies } \\
(\mathrm{MHz})\end{array}$ & $\begin{array}{c}\text { obs.-calc. } \\
(\mathrm{MHz})\end{array}$ \\
\hline 13 & $\mathbf{9 8 5 5 . 2 5}$ & 9855.30 & -0.05 \\
14 & 11370.44 & 11370.41 & +0.03 \\
15 & 12993.43 & 12993.41 & +0.02 \\
16 & 14724,27 & 14724.23 & +0.04 \\
17 & 16562.80 & 16562.83 & $-0,03$ \\
18 & 18509.00 & 18509.05 & $-0,05$ \\
19 & 20562.96 & 20562.90 & +0.06 \\
20 & 22724.31 & 22724.23 & +0.08 \\
21 & 24992.97 & 24992.97 & 0.00 \\
22 & 27368.98 & 27369,02 & -0.04 \\
23 & 29852.22 & 29852.25 & -0.03 \\
24 & 32442.59 & 32442.60 & -0.01 \\
25 & 35139.95 & 35139.93 & +0.02 \\
\hline
\end{tabular}

Table 3. Direct $l$-type doublet transitions measured for the $0000^{0} 3^{1}$ state of $\mathrm{H}^{12} \mathrm{C}^{14} \mathrm{~N}^{16} \mathrm{O}$. The calculated frequencies were obtained by using the constants given in Table 4, Method II.

existing correlations between $q_{5}^{(2)}$ and $\Delta$ which would lead to a system of normal equations ill-conditioned in these parameters. Therefore the analysis was carried out both

1) with $q_{5}^{(2)}=0$, and

2) with the assumption that

$$
q_{3 \times 5}^{(2)}=q_{1 \times 5}^{(2)}=1 \cdot 10^{-9} \mathrm{MHz} \text {. }
$$

The results of both procedures are presented in Table 4. The numerical values of $q^{(0)}$ and $q^{(1)}$ agree for both mehods within the calculated error limits. The standard deviation of the observed lines is within the estimated experimental error of \pm 0.05 $\mathrm{MHz}$ in both cases. However, the second method is considered more reliable since the value of $\Delta$ is $30 \%$ more accurate and because the assumption $q_{3 \times 5}^{(2)}=q_{1 \times 5}^{(2)}$ is physically more meaningful than the assumption $q_{3 \times 5}^{(2)}=0$. The calculated line frequencies in Table 3 were obtained using the constants of method 2. For a more detailed description of the error definitions see Ref. ${ }^{1}$.

Our measurements suggest neither any $J$-dependence of the constant $\Delta$ nor the need of including matrix elements of the type

$$
\left(v_{\mathrm{t}}, l_{\mathrm{t}}\left|H^{(2)^{\prime}}\right| v_{\mathrm{t}}, l_{\mathrm{t}} \pm 4\right)
$$

in the derivation of the frequency expression.

The magnitude of $q_{3 \times 5}^{(0)}$ and $\Delta$ suggest that measurements of the splitting of the $\Phi$ levels will be difficult to obtain, since the direct doublet transitions

12 H. K. Bodenseh and M. Winnewisser, Z. Naturforsch. 24 a, 1973 [1969]. 


\begin{tabular}{|c|c|c|c|c|}
\hline & $\begin{array}{c}q_{3 \times 5}^{(0)} \\
(\mathrm{MHz})\end{array}$ & $\begin{array}{c}q_{3 \times 5}^{(1)} \\
(\mathrm{MHz})\end{array}$ & $\stackrel{4}{4}$ & $\begin{array}{c}\text { Standard } \\
\text { deviation of fit. }\end{array}$ \\
\hline $\begin{array}{l}\text { Method I } \\
q_{3 \times 5}^{(2)}=0\end{array}$ & $27.09201 \pm 1.6 \cdot 10^{-4}$ & $(9.255 \pm 0.073) \cdot 10^{-5}$ & $(2.30 \pm 0.33) \cdot 10^{6}$ & 0.041 \\
\hline $\begin{array}{l}\text { Method II } \\
q_{3 \times 5}^{(2)}=q_{1 \times 5}^{(2)}=1 \cdot 10^{-9}\end{array}$ & $27.09196 \pm 1.8 \cdot 10^{-4}$ & $(9.238 \pm 0.082) \cdot 10^{-5}$ & $(1.95 \pm 0.22) \cdot 10^{6}$ & 0.046 \\
\hline
\end{tabular}

Table 4. l-type doubling constants calculated for the $0000^{0} 3^{1}$ vibrational state of $\mathrm{H}^{12} \mathrm{C}^{14} \mathrm{~N}^{16} \mathrm{O}$.

for $J=100$ fall in the $5 \mathrm{GHz}$ region. The difference of the splitting for two adjacent $J$-values ( $l$-type doubling of normal R-branch rotational transitions) is only $0.05 \mathrm{MHz}$ for the $J=16 \rightarrow 17$ transition at $400 \mathrm{GHz}$.

Considering the results of the foregoing analysis in comparison with analogous results for $v_{5}=1$ (Ref. ${ }^{1}$ ) we find that the doubling constant $q_{5}{ }^{(0)}$ changes from $34.64 \mathrm{MHz}$ for $v_{5}=1$ to $27.09 \mathrm{MHz}$ for $v_{5}=3$, whereas it is expected to be a constant valid for both states. In addition, the constant $\Delta$ representing the energy difference between the $\Pi$ and $\Phi$ levels in the $v_{5}=3$ state has the rather large value of $1.95 \cdot 10^{6} \mathrm{MHz}=65.0 \mathrm{~cm}^{-1}$, a factor of 2 larger than the corresponding value for $\mathrm{HCN}^{5}$.

Both effects are somewhat surprising. The change in $q$ from $v_{5}=1$ to $v_{5}=3$ can be attributed to a vibrational dependence similar to that of the rotational constant as given in Eq. (2). However, the change in $q$ is too large for such an explanation to be fully satisfactory. Again it is necessary to fall back on the posibilty of resonance interactions to account for the value of $q_{(3 \times 5)}^{(0)}$. As can be seen from Fig. 3 an interaction of the Darling-Dennison type may connect the vibrational state $0000^{0} 3^{3,1}$ with the state $000(21)^{3,1}$, and such an interaction would certainly perturb the constant $q$.

Since the matrix elements for the Darling-Dennison type second order perturbation depend on $l$ [see Eq. (16) ] the perturbation will not affect the $\Pi$ and $\Phi$ levels of the $0000^{0} 3^{3,1}$ state equally. The "unperturbed" energy levels $E_{3}{ }^{0}$ and $E_{1}{ }^{0}$ used in the secular equation to derive the $l$-type doubling frequencies are therefore actually already perturbed by a second order interaction of this type. The large $\Delta=E_{3}{ }^{0}-E_{1}{ }^{0}$ found may most probably be accounted for in this way. In addition contributions from second order anharmonic resonance interactions having matrix elements of the type

$$
\left(v_{\mathrm{t}}, v_{\mathrm{t}^{\prime}}, v_{\mathrm{t}^{\prime \prime}}\left|H^{(2)^{\prime}}\right| v_{\mathrm{t}} \pm 1, v_{\mathrm{t}^{\prime}} \mp 1, v_{\mathrm{t}^{\prime \prime}}\right) \text { etc. }
$$

and having force constants of the type $k_{n n 45}$ cannot be completely ruled out.

A quantitative calculation of the magnitude of the resonance interactions among the observed levels requires the diagonalization of the energy matrix for an entire polyad, that is, the entire group of levels connected by first or second order interactions, including $l$-type phenomena. This will not be possible until sufficient infrared and millimeter wave data become available.

\section{Acknowledgements}

We express our appreciation to Professor Dr. WERNER ZEIL for the possibility of conducting this research in his laboratory. The work was supported by the Deutsche Forschungsgemeinschaft. The calculations were performed on the IBM 7094 computer of the Deutsches Rechenzentrum in Darmstadt. The use of library programs is gratefully acknowledged. The authors are indebted to Dr. WOLfGANG HÜTTNER and Dr. BRENDA WINNEWISSER for helpful discussions and suggestions. 